\title{
Novel Selected Modes on The Conductor-Backed Slotline
}

\author{
Jan Zehentner, Jan Machac, and Jan Mrkvica
}

Czech Technical University, Technicka 2, 16627 Prague 6, Czech republic

\begin{abstract}
This paper presents a detailed full-wave analysis of the conductor-backed slotline. The occurrence of an even dominant mode is revealed. This mode may propagate alone from de up to the frequency at which the first leaky mode sets up. Nonphysical solutions of the dispersion equation and also the second leaky mode, taking power in the substrate, are identified. The first odd space leaky mode revealed on the CBSL enable an entirely uniplanar leaky wave antenna to be designed. The dispersion characteristics of particular revealed modes and their field distributions on the cross-sectional plane are also presented.
\end{abstract}

\section{INTRODUCTION}

Printed-circuit lines have become the standard transmission lines in today's MIC, in both the micro-wave and the mm-wave bands. During the last thirty years hundreds of works have discussed their properties, their behaviour and the modifications most suitable for particular applications. Until now the conductor-backed slotline (CBSL) has stood at the margin of the interest of designers, apparently due to the power leakage into the substrate, and only a few publications [1]-[3] have worked on analysing it. Our present work attempts to find out whether the CBSL can act as a radiator, being a counterpart to the microstrip leaky wave antenna [4], radiating only to half-space.

This paper presents a full-wave analysis of the CBSL. First, we compare our results with those available in the literature. Then we present for the first time the dispersion characteristics of the even modes of the narrow and odd mode of the wide CBSL, i. e., the dominant mode, modes leaking into the substrate, and the space leaky mode. Finally, the calculated and measured propagation constant and the field distribution of selected modes will clarify the propagation and power leakage of the constitutive modes occurring on the laterally and longitudinally unbounded CBSL.

The analysis brings novel findings, a better understanding of CBSL behaviour, and will facilitate designing the CBSL to meet desired application requirements. This pushes forward the general view on the ability of CBSL to guide or radiate waves. A suitable design can then eliminate undesired couplings in the circuit, or can support anticipated space radiation.

\section{COMPARISON OF ALREADY KNOWN AND NEWLY PRESENTED LEAKY MODE CHARACTERISTICS OF THE CONDUCTOR-BACKED SLOTLINE}

The conductor-backed coplanar waveguide (CBCPW) and the conductor-backed slotline enable location of circuits on a metal base, ensuring improved mechanical strength, good heat sinking and easy dc biasing. Their great drawback is a leakage of power in the substrate in the form of the parallel-plate TEM wave, resulting in undesired coupling to the neighbouring circuit sections. Several concepts for suppressing this leakage have been proposed and successfully tested [5]-[7]. On the other hand, we assume that CBSL could radiate in the half-space and take full advantage of planar circuit, feeder and antenna integration. We are therefore investigating the lossless, laterally and longitudinally unbounded CBSL, Fig. 1, in the spectral domain approach.

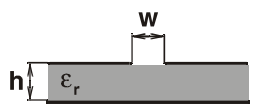

Fig. 1. Cross-section of a conductor-backed slotline.

The propagation constant, i. e., the phase and leakage constant of the 1st leaky wave, carrying power into the substrate, normalized to the propagation constant of free space, was calculated by the mode matching method applied to the CBSL in [1]. A line on the substrate with permittivity 2.25 had the top cover $12.5 \mathrm{~h}$ from the upper line surface. According to [1] the height of the cover plate has a minor influence on the propagation constant. The propagation constant of the CBSL with dielectric permittivity 2.55 was calculated in the spectral domain [3], where $\mathrm{h} / \lambda_{0}=0.267$ was chosen, as in [1]. A comparison of the propagation constants calculated according to [1] and [3] in Fig. 11 of [3] for different permittivity is therefore rather questionable.

Based on the method of moments applied in the spectral domain we produced a code calculating the propagation constant of a selected mode on the CBSL. The second code calculates the electric field distribution of that mode on the cross-sectional plane by means of the backward Fourier transform. Since the only available relevant values are in [1] and [3], we computed the propagation constant of the 1st leaky wave on the CBSL, setting permittivity 
2.25, and plotted it in Fig. 2, where for comparison corresponding data taken over from [1] and [3] are also drawn. Two basis functions for the transversal and for the longitudinal components of the electric field were used. A greater number of basis functions did not significantly change the propagation constant. Phase constant $\beta / \mathrm{k}_{0}$ obtained by these three approaches differs more or less according to $w / \lambda_{0}$. A better fit, except in the low frequency region, is found with $\alpha / \mathrm{k}_{0}$. In any case, the shapes of the dispersion characteristics resemble each other well. Consequently we continue to consider our code sound and reliable.
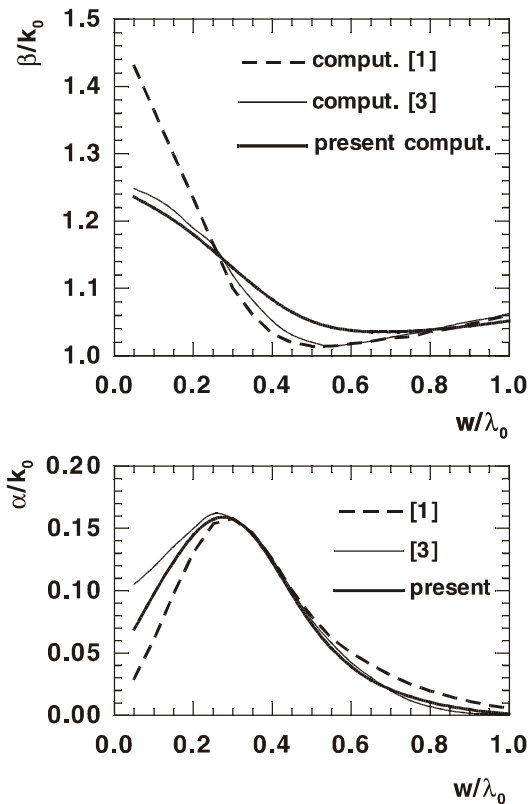

Fig. 2. Normalized phase and leakage constant of the 1st leaky wave on the CBSL computed in [1] and [3] compared with our values.

\section{NEW DisPERSION CHARACTERISTICS OF THE CONDUCTOR-BACKED SLOTLINE}

On a transmission line consisting of three conductors placed in a non-homogeneous dielectric, as is the CBSL case, two dominant hybrid or quasi TEM waves can propagate when the frequency increases from zero. These waves have even and odd symmetry of the transversal electric field component within the slot with regard to the plane of the line symmetry. In this paper we inquire into the even modes. The odd modes are discussed only when space leakage is considered.

The propagation constants of the CBSL with a slot comparable to the substrate thickness, depending on the normalized frequency $h / \lambda_{0}$, are shown in Fig. 3. Their appearance changes dramatically with a change in crosssection size and permittivity. This issue goes beyond the scope of this paper.
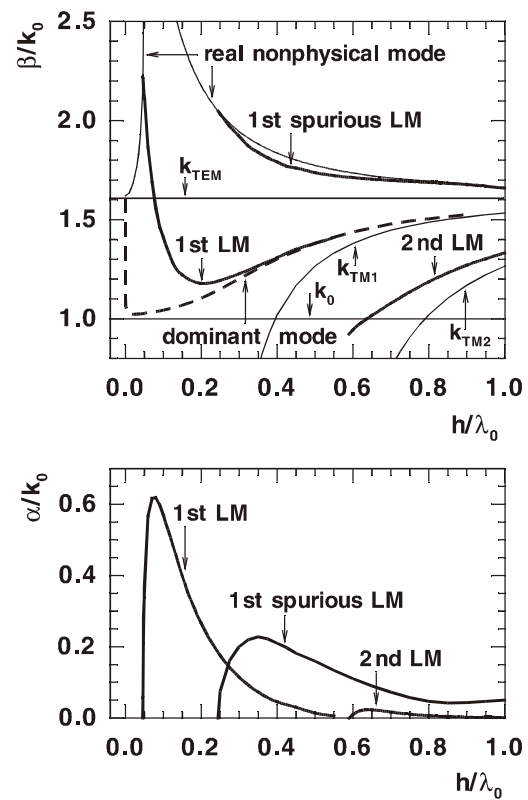

Fig. 3. Normalized dispersion characteristics of the even dominant and even leaky modes on the CBSL with $\mathrm{w}=6 \mathrm{~mm}$, $\mathrm{h}=6 \mathrm{~mm}, \varepsilon_{\mathrm{r}}=2.6$.

A typical transmission line, e. g., a microstrip line, conductor-backed strips or optical fibre, guides waves along the boundary of its higher effective permittivity core area encompassed by the outer lower effective permittivity area. However, the CBSL has an inverted effective permittivity arrangement. The central area, under the slot, has lower effective permittivity and the outer area, between the parallel plates, has higher effective permittivity, which equals $\varepsilon_{\mathrm{r}}$. Nevertheless, the even and odd dominant modes do exist. These modes are not typical bound modes with the power flow dominating in the central area and the amplitude monotonically decreasing laterally. We will refer to them as the even and odd dominant modes.

The even dominant mode starts at zero frequency. It has only a real phase constant, which tends from 0 to $\mathrm{k}_{\mathrm{TEM}}=\sqrt{ } \varepsilon_{\mathrm{r}} \mathrm{k}_{0}$, the propagation constant of the TEM parallelplate mode, and can unattenuatedly propagate along the line by oneself. Its propagation constant computed in the full-wave approach holds even for decreasing frequency down to dc, see Fig. 4, a zoomed part of Fig. 3 with removed normalization. The even dominant mode has the standing wave feature perpendicular to the line axis with laterally constant magnitude, a consequence of the solution of the dispersion equation of the unbounded sourceless line. 


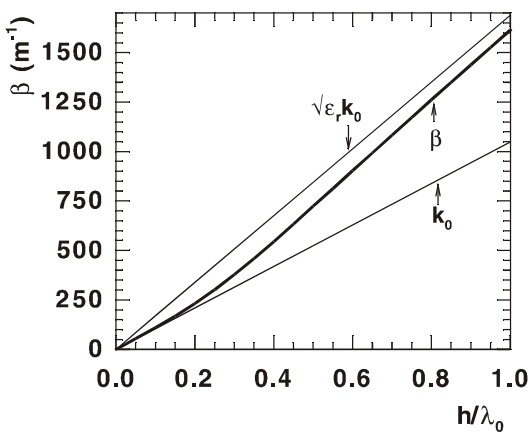

Fig. 4. Phase constant of the even bound wave on the CBSL when $\mathrm{w}=6 \mathrm{~mm}, \mathrm{~h}=6 \mathrm{~mm}, \varepsilon_{\mathrm{r}}=2.6$

Since we are inquiring into the ideal unbounded CBSL, the occurrence of a lateral standing wave accompanying the dominant mode can be explained as follows. Two parallel-plate modes are incident at an angle from the left and right on the central part of the CBSL where the effective permittivity is less than $\varepsilon_{\mathrm{r}}$. At this non-sharp boundary they partly reflect back to the area with $\varepsilon_{\mathrm{r}}$ producing a standing wave, and partly excite so called "surface waves" in the central area of the line. Each of these "surface waves" propagates along its virtual boundary, i. e., parallel to the line axis, and transports power. An alternative way of forming a standing wave assumes a wave incident from above, perpendicular to the slot with a crosswise slot electric field orientation. Now two waves propagate laterally between parallel plates towards the opposite infinity. Each wave returns through infinity to the opposite slot edge and adds up with the second counter directionally propagating particular wave, resulting in a rise of the standing wave. Fig. 5 plots the electric field distribution of the even dominant mode on the cross-sectional plane.

In Fig. 3 we have two branches of the real nonphysical solution of the dispersion equation which join at great $\beta / \mathrm{k}_{0}$. The wider the slotwidth the lower is this meeting point. The first leaky mode characteristic splits off from the real nonphysical solution. This mode becomes a physical wave at frequencies when its phase constant falls below $\mathrm{k}_{\mathrm{TEM}}$. We therefore have a frequency band in which the only dominant mode propagates. However, there is also a spurious leaky mode, i. e., a complex solution which is nonphysical since its $\beta$ is greater than $\mathrm{k}_{\mathrm{TEM}}$ and splits off again from the real nonphysical solution. When the slotwidth grows, these split-off points of the true and spurious 1st leaky modes merge and become identical when $w=7.001375 \mathrm{~mm}$ for the CBSL studied here. Far above this critical slotwidth the spurious solution becomes physical and we have an ambiguous 1st leaky mode solution with significantly different propagation constants.
The second leaky mode, carrying power as the TEM parallel-plate mode and simultaneously as the first $\mathrm{TM}_{1}$ mode, appears at higher frequencies. It converts to a physical leaky wave when its phase constant is greater than $\mathrm{k}_{0}$, and remains less than $\mathrm{k}_{\mathrm{TM} 1}$.

The even mode spectrum of the CBSL contains no bound mode. The CBSL is not suitable for power transport. At the lowest frequencies there is the even dominant mode which can couple to neighbouring circuits, the mechanism of which differs from leakage. At higher frequencies this dominant mode and the 1st leaky mode can propagate simultaneously and their phase constants merge. They may appear to be coupled, but they are still physical and still differ.

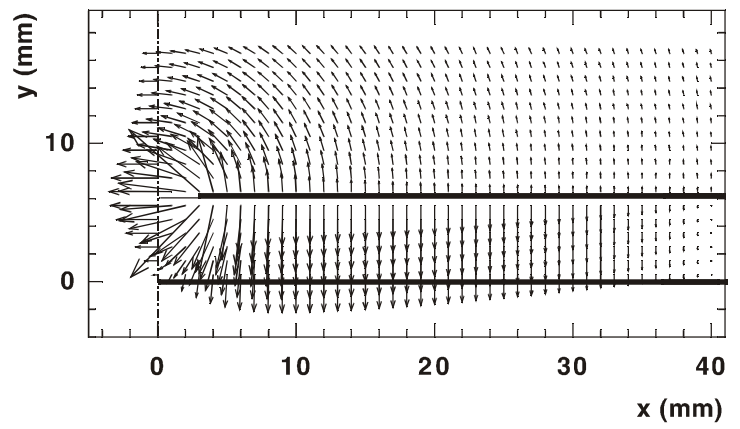

Fig. 5. Electric field of the even dominant mode at the cross sectional plane when $\mathrm{w}=6 \mathrm{~mm}, \mathrm{~h}=6 \mathrm{~mm}, \varepsilon_{\mathrm{r}}=2.6$ and $\mathrm{h} / \lambda_{0}=0.03$.

The electric field distribution of the 1st leaky wave on the cross-sectional plane is plotted in Fig. 6. We measured the electric field along the slot of the CBSL and perpendicular to its axis at the edge of the substrate in the set-up as used in [1]. Fig.7 shows a good agreement between the calculated and measured 1st leaky wave propagation constants.

\section{SPACE LEAKy Mode ON THE CONDUCTOR-BACKed SLOTLINE}

The field in a sufficiently wide slot of the CBSL excites a radiated field. We identified the even space leaky modes when $\mathrm{h} / \lambda_{0}$ in Fig. 3 moves roughly from 0.08 to 0.55 . However, it is questionable what kind of source would excite the proper field in the slot. Consequently we turned our attention to the odd modes. The first odd, frequency lowest, space leaky mode characteristic of the CBSL with a "wide" slot is shown in Fig. 8. The mode is physical when its phase constant is less than $\mathrm{k}_{0}$. The relatively great leakage constant calls forth a doubt about the radiation efficiency. Nevertheless this leaky mode should radiate one main beam in the half-space. Its field in the neighbourhood of the slot is plotted in Fig. 9. 


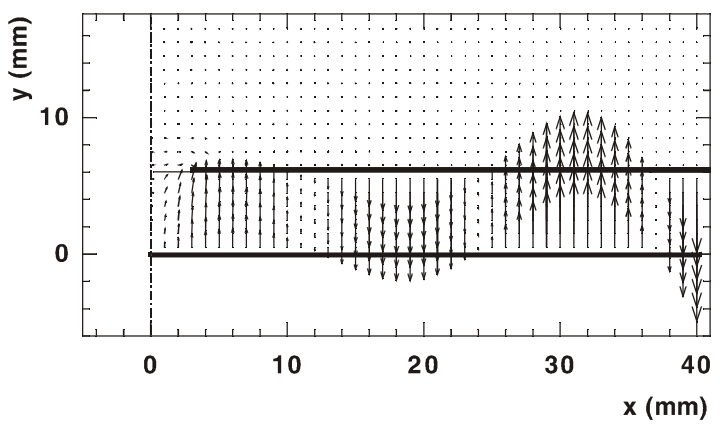

Fig. 6. Electric field of the 1st leaky mode on the crosssectional plane when $\mathrm{w}=6 \mathrm{~mm}, \mathrm{~h}=6 \mathrm{~mm}, \varepsilon_{\mathrm{r}}=2.6$ and $\mathrm{h} / \lambda_{0}=0.2$.

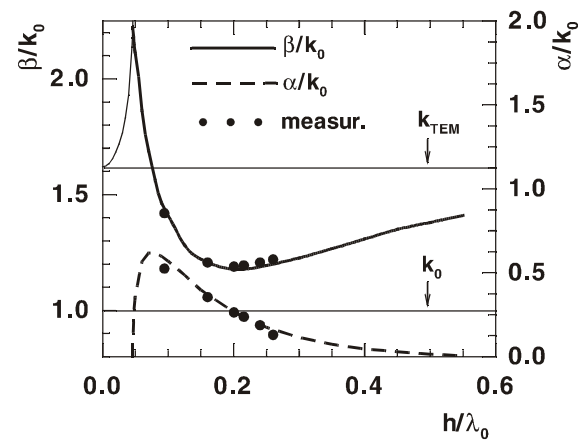

Fig. 7. Calculated and measured phase and leaky constants of the $1 \mathrm{st}$ leaky mode on the CBSL with $\mathrm{w}=6 \mathrm{~mm}, \mathrm{~h}=6 \mathrm{~mm}, \varepsilon_{\mathrm{r}}=2.6$.

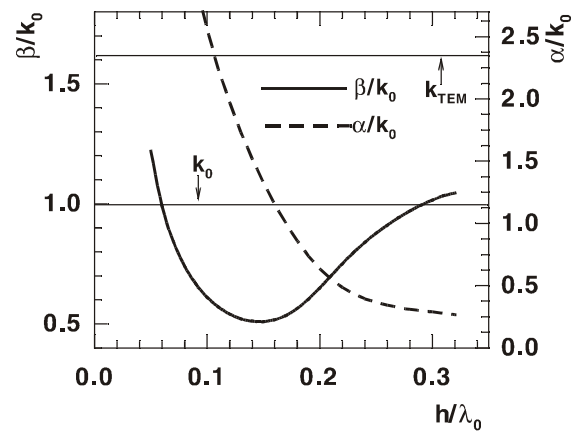

Fig. 8. Propagation constant of the space leaky mode on the CBSL with $\mathrm{w}=20 \mathrm{~mm}, \mathrm{~h}=8 \mathrm{~mm}, \varepsilon_{\mathrm{r}}=2.6$.

\section{CONCLUSIONS}

A full-wave analysis shows that the even dominant mode may propagate on the CBSL alone from dc to the frequency at which the first leaky mode sets up. The field of this dominant mode in the substrate outside the close vicinity of the slot has a standing wave behaviour perpendicular to the line axis. The calculated and measured propagation constants of the 1st leaky wave in the delimited frequency band compare well. The second even leaky mode occurs at very high frequencies. On the wide slotted CBSL and at high frequencies the field may simultaneously consist of at least three meaningful modes: the dominant mode producing a standing wave laterally, the 1st double degenerated leaky mode with laterally increasing magnitude, and the 2nd leaky mode also twice degenerated. The real nonphysical solutions of the dispersion equation were identified. They and also the 1 st leaky mode solution change rapidly with line size and substrate permittivity. The first odd space leaky mode may occur on the CBSL with a wide slot. This finding offers an opportunity for designing a leaky wave antenna. Unlike lateral unbounded CBSL is not suitable for power transmission.

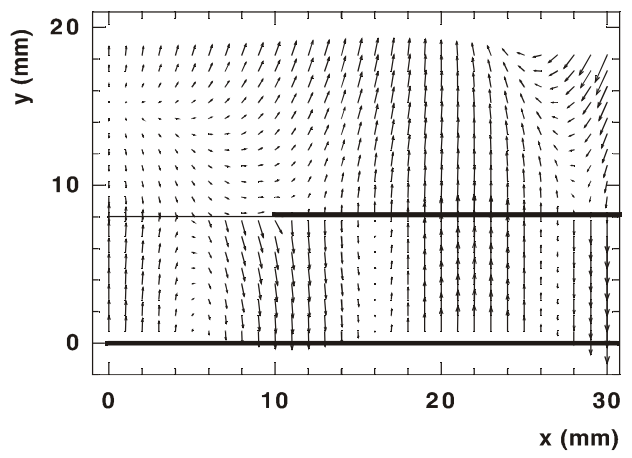

Fig. 9. Electric field of the first odd space leaky mode on the CBSL with $\mathrm{w}=20 \mathrm{~mm}, \mathrm{~h}=3 \mathrm{~mm}, \varepsilon_{\mathrm{r}}=2.6$ and $\mathrm{h} / \lambda_{0}=0.28$.

\section{REFERENCES}

[1] H. Shigesawa, M. Tsuji, and A. A. Oliner, "Conductorbacked slot line and coplanar waveguide: Dangers and fullwave analysis," 1988 IEEE MTT-S Int. Microwave Symp. Dig., vol. 1, pp. 199-202, June 1988.

[2] H. Shigesawa, M. Tsuji, and A. A. Oliner, "Dominant mode power leakage from printed-circuit waveguides," Radio Science, vol. 26, no. 2, pp. 559-564, March-April 1991.

[3] N. K. Das, D. M. Pozar, "Full-wave spectral-domain computation of material, radiation, and guided wave losses in infinite multilayered printed transmission lines," IEEE Trans. Microwave Theory and Tech., vol. MTT-39, no. 1, pp. 54-63, January 1991.

[4] W. Menzel, "A new travelling-wave antenna in microstrip", Archiv fur Ubertragungstechnik, vol. 33, no. 4, pp. 137140, April 1979.

[5] Y. Liu, K. Cha, and T. Itoh, "Non-leaky coplanar (NLC) waveguides with conductor backing," IEEE Trans. Microwave Theory and Tech., vol. MTT-43, no. 5, pp. 1067-1072, May 1995.

[6] N. K. Das, "Methods of suppression or avoidance of parallel-plate power leakage from conductor-backed transmission lines," IEEE Trans. Microwave Theory and Tech., vol. MTT-44, no. 2, pp. 169-181, Februaryy 1996.

[7] K-P. Ma, F-R. Yang, et al, "Nonleaky conductor-backed CPW using a novel 2-D PBG lattice," 1998 Asia-Pacific Microwave Conference Proceedings, vol. 2, pp. 509-512, December 1998. 